\title{
The Investigation of MHD Williamson Nanofluid over Stretching Cylinder with the Effect of Activation Energy
}

\author{
Wubshet Ibrahim $\mathbb{D}^{1}$ and Mekonnen Negera ${ }^{2}$ \\ ${ }^{1}$ Department of Mathematics, Ambo University, Ambo, Ethiopia \\ ${ }^{2}$ Department of Mathematics, Wollega University, Nekemte, Ethiopia \\ Correspondence should be addressed to Wubshet Ibrahim; wubshetib@yahoo.com
}

Received 15 March 2020; Revised 18 May 2020; Accepted 3 June 2020; Published 25 June 2020

Academic Editor: Emilio Turco

Copyright ( 2020 Wubshet Ibrahim and Mekonnen Negera. This is an open access article distributed under the Creative Commons Attribution License, which permits unrestricted use, distribution, and reproduction in any medium, provided the original work is properly cited.

\begin{abstract}
In this paper, we discussed the effect of activation energy on mixed convective heat and mass transfer of Williamson nanofluid with heat generation or absorption over a stretching cylinder. Dimensionless ordinary differential equations are obtained from the modeled PDEs by using appropriate transformations. Numerical results of the skin friction coefficient, Nusselt number, and Sherwood number for different parameters are computed. The effects of the physical parameter on temperature, velocity, and concentration have been discussed in detail. From the result, it is found that the dimensionless velocity decreases whereas temperature and concentration increase when the porous parameter is enhanced. The present result has been compared with published paper and found good agreement.
\end{abstract}

\section{Introduction}

The heat and mass transfer of boundary layer flow of nonNewtonian fluids over a stretching cylinder is of great interest for scientists, engineers, and researchers due to its wide applications. Some examples of its applications are the extrusion process, extraction of metals, annealing, thinning of copper wire, and pipe industry. Nowadays, in most industries, the significance of non-Newtonian fluids governs the Newtonian fluids. The rheological properties of non-Newtonian fluids cannot be illuminated by the classical Naiver-Stokes equations. Also, non-Newtonian fluid characteristics cannot be modeled by a single model. To overcome this difficulty, ample models have come into being. The rheological models that were projected were Williamson, Cross, Ellis, power law, Carreau fluid model, etc. Typical of a non-Newtonian fluid model with shear retreating property is the Williamson fluid model and was first expected by Williamson [1]. Some recent investigations concerning the flow and heat and mass transfer of MHD flow of Williamson fluid can be mentioned through investigations [2-6].
Heat and mass transfer in non-Newtonian fluid flow through porous medium in engineering has extensive application, such as ventilation procedure, oil production, solar collection, cooling of nuclear reactors, and electronic cooling. Accordingly, Maripala and Kishan [7] investigated the effect of thermal radiation and chemical reaction on timedependent MHD flow and heat transfer of nanofluid over a permeable shrinking sheet. Their result indicates that with an increase of the suction parameter, the temperature profiles decrease whereas the concentration profiles upsurge. Moreover, Kairi and RamReddy [8], Vijaya et al. [9], Ambreen et al. [10], Aurangzaib et al. [11], Bal Reddy et al. [12], Al-Mamun et al. [13], and Ahmed et al. [14] studied mixed convective heat and mass transfer of MHD nanofluids embedded in porous medium over stretching surfaces. From their result, it can be seen that temperature distribution and thermal boundary layer reduce with an increase of dimensionless thermal free convection parameter, dimensionless mass free convection parameter, and Prandtl number.

The miracle of heat generation/absorption in working fluid plays an important role in several engineering and 
industries. The influence of heat generation may increase the temperature distribution in moving fluids and consequently impacts the heat transfer rate. Some of its application in many industries is the geothermal system, thermal absorption, removal of heat from nuclear wreckage, food storage, microelectronics manufacturing, and cooling of nuclear reactors. Consequently, heat generation or absorption in MHD flow of Casson fluid over a stretching wedge with viscous dissipation and Newtonian heating was investigated by Ullah et al. [15]. Moreover, Khan et al. [16] and Padmavathi and Anitha Kumari [17] examined the effect of heat generation or absorption on mixed heat and mass transfer nanofluids embedded over stretching surfaces. Some researchers have studied the impact of heat source/sink on MHD nonNewtonian nanofluids over stretching surfaces [18-23].

Activation energy is the smallest amount of energy needed by chemical reactants to endure a chemical reaction. The influence of activation energy on convective heat and mass transfer in the region of boundary layers was initially inspected by Bestman [24]. Later, many researchers have studied the impact of activation energy on heat and mass transfer of boundary layer flow of the fluids. Among those researchers, Awad et al. [25], Dhlamini et al. [26], Anuradha and Sasikala [27], and Hamid et al. [28] scrutinized the influence of activation energy on heat and mass transfer in unsteady fluid flow under different geometry. Moreover, Huang [29] and Mustafa [30] studied the effect of activation energy on MHD boundary layer flow of nanofluids past a vertical surface and permeable horizontal cylinder. Further investigations on the effect of activation energy on nonNewtonian fluid under different surfaces are stated in [31-34]. From their plots, it can be seen that as the values of the activation energy parameter upsurges, the concentration nanoparticles increase.

In view of all the above-revealed studies, it is decided that the effect of activation energy on mixed convective heat and mass transfer of Williamson nanofluid over a stretching cylinder embedded in a porous medium with heat generatio$\mathrm{n} /$ absorption is not examined yet. Thus, to fill this gap, we aim to explore the effect of activation energy on mixed convective heat and mass transfer of Williamson nanofluid over a stretching cylinder embedded in a porous medium with heat generation/absorption. For information, our work is innovative in terms of the proposed fluids and incorporated parameters in the boundary layer flow. A numerical solution is obtained by using the Runge-Kutta method in conjugation with the shooting technique. Numerical results of the Nusselt number, skin friction coefficient, and Sherwood number for different values of the physical parameter are computed in tables. The effects of different physical parameters on dimensionless velocity, temperature, and concentration are presented in graphs.

\section{Mathematical Formulation}

Two-dimensional time-independent Williamson nanofluid over a stretching cylinder in the presence of a boundary layer slip has been considered. A porous cylinder is chosen as shown in Figure 1. The magnetic field of constant strength

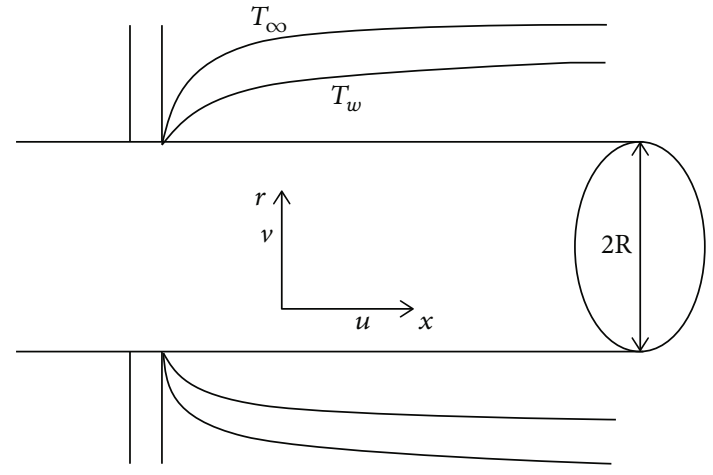

Figure 1: Physical model of the problem.

$B_{0}$ is applied normal to the flow along the $r$-axis in a radial direction with the assumption of a small Reynolds number so that the induced magnetic field is neglected. The fluid is infinite in magnitude of positive $x$-direction. The mixed convective mass and heat transfer phenomenon are included in the existence of heat generation/absorption. $T_{w}$ and $C_{w}$ are surface temperature and concentration, respectively. The ambient fluid temperature and concentration are denoted by $T_{\infty}$ and $C_{\infty}$, respectively. Further, $u$ and $v$ are the velocity components in the direction of $x$ and $r$, respectively. All the physical properties of the fluid are considered constant.

Using the assumptions given above, the governing equations of continuity, momentum, energy, and mass are specified as follows:

$\frac{\partial}{\partial x}(r u)+\frac{\partial}{\partial y}(r v)=0$

$$
\begin{aligned}
u \frac{\partial u}{\partial x}+v \frac{\partial u}{\partial r}= & v\left(\frac{1}{r} \frac{\partial u}{\partial r}+\frac{\partial^{2} u}{\partial r^{2}}\right) \\
& +v\left(\frac{\Gamma}{\sqrt{2} r}\left(\frac{\partial u}{\partial r}\right)^{2}+\sqrt{2} \Gamma \frac{\partial u}{\partial r} \frac{\partial^{2} u}{\partial r^{2}}\right)-\frac{v}{k^{\prime}} u \\
& -\frac{\sigma_{1} B_{0}^{2}}{\rho} u+g \beta_{t}\left(T-T_{\infty}\right)+g \beta_{c}\left(C-C_{\infty}\right) \\
= & 0,
\end{aligned}
$$

$$
\begin{aligned}
u \frac{\partial T}{\partial x}+v \frac{\partial T}{\partial r}= & \frac{k}{\rho c_{p}}\left(\frac{1}{r} \frac{\partial T}{\partial r}+\frac{\partial^{2} T}{\partial r^{2}}\right) \\
& +\tau\left\{D_{B} \frac{\partial C}{\partial r} \frac{\partial T}{\partial r}+\frac{D_{T}}{T_{\infty}}\left(\frac{\partial T}{\partial r}\right)^{2}\right\} \\
& +\frac{Q}{\rho c_{p}}\left(T-T_{\infty}\right),
\end{aligned}
$$




$$
\begin{aligned}
u \frac{\partial C}{\partial x}+v \frac{\partial C}{\partial r}= & D_{B}\left(\frac{1}{r} \frac{\partial C}{\partial r}+\frac{\partial^{2} C}{\partial r^{2}}\right)+\frac{D_{T}}{T_{\infty}}\left(\frac{1}{r} \frac{\partial T}{\partial r}+\frac{\partial^{2} T}{\partial r^{2}}\right) \\
& -k_{r}^{2}\left(C-C_{\infty}\right)\left(\frac{T}{T_{\infty}}\right)^{e} \exp \left(\frac{E_{a}}{k_{1} T}\right) .
\end{aligned}
$$

The boundary conditions are

$$
\begin{aligned}
& u=u_{0} \frac{x}{L}+b_{0} v \frac{\partial u}{\partial r}, \\
& v=-v_{w}, \\
& T=T_{w}, \\
& C=C_{w} \text { at } r=R, \\
& u \longrightarrow u_{\infty}, \\
& T \longrightarrow T_{\infty}, \\
& C \longrightarrow C_{\infty}, \text { as } r \longrightarrow \infty,
\end{aligned}
$$

where $T_{w}=T_{\infty}+T_{0}(x / L)^{\beta}$ and $C_{w}=C_{\infty}+C_{0}(x / L)^{\varsigma}$ are surface temperature and surface concentration, respectively; $u_{0}$ , $T_{0}$, and $C_{0}$ are reference velocity, temperature, and concentration, respectively; $\beta$ is temperature exponent; and $\varsigma$ is concentration exponent signifying the change of amount of solute in $x$-direction.

The partial differential equations given in equations (2)-(4) are converted to a system of ordinary differential equations by inspiring the following similarity transformations:

$$
\begin{aligned}
\eta & =\frac{r^{2}-R^{2}}{2 R}\left(\frac{u_{0}}{x v}\right)^{1 / 2}, \\
\theta(\eta) & =\frac{T-T_{\infty}}{T_{w}-T_{\infty}}, \\
\phi(\eta) & =\frac{C-C_{\infty}}{C_{w}-C_{\infty}}, \\
u & =\frac{1}{r} \frac{\partial \psi}{\partial r}, \\
v & =-\frac{1}{r} \frac{\partial \psi}{\partial x} .
\end{aligned}
$$

Thus, the system of ordinary differential equations is

$$
\begin{aligned}
(1+2 \eta A) f^{\prime \prime \prime} & +2 A f^{\prime \prime}+\frac{3}{2}(1+2 \eta A)^{1 / 2} \lambda f^{\prime \prime 2} \\
& +\lambda(1+2 \eta A)^{3 / 2} f^{\prime \prime} f^{\prime \prime \prime}-f^{\prime 2}-(d+M) f^{\prime} \\
& +p \theta+q \phi=0,
\end{aligned}
$$

$$
\begin{aligned}
(1+2 \eta A) \theta^{\prime \prime} & +\operatorname{PrNb}(1+2 \eta A) \theta^{\prime} \phi^{\prime}+2 A \theta^{\prime} \\
& +\operatorname{Pr} f^{\prime} \theta^{\prime}+\operatorname{PrNt}(1+2 \eta A) \theta^{\prime 2} \\
& +\operatorname{Pr} S \theta-\operatorname{Pr} \beta f^{\prime} \theta=0, \\
(1+2 \eta A) \phi^{\prime \prime}+\operatorname{Sc} f \phi^{\prime} & +2 A \phi^{\prime}+2 \frac{\mathrm{Nt}}{\mathrm{Nb}} A \theta^{\prime}+\frac{\mathrm{Nt}}{\mathrm{Nb}}(1+2 \eta A) \theta^{\prime \prime} \\
- & \mathrm{Sc} \sigma(1+\zeta \theta)^{e} \exp \left(\frac{-E}{1+\zeta \theta}\right)-\operatorname{Scc} f^{\prime} \phi=0 .
\end{aligned}
$$

With the boundary conditions

$$
\begin{aligned}
f(0) & =\delta, \\
f^{\prime}(0) & =1+\gamma f^{\prime \prime}(0), \\
\theta(0) & =1, \\
\phi(0) & =1, \\
f^{\prime}(\infty) & =0, \\
\theta(\infty) & =0, \\
\phi(\infty) & =0,
\end{aligned}
$$

where $M=\left(\sigma_{1} B_{0}^{2} L / \rho u_{0}\right)^{1 / 2}$ is the magnetic parameter, $A=$ $\sqrt{v L / u_{0} R^{2}}$ is the curvature parameter, $\gamma=B_{0} \sqrt{v u_{0} / L}$ is the slip parameter, $\lambda=\Gamma \sqrt{2 u_{0}^{3} / v L}$ is the Weissenberg number (Williamson parameter), $p=g \beta_{t}\left(T_{w}-T_{\infty}\right) / \operatorname{Re}_{x} v$ is the local temperature buoyancy parameter, $q=g \beta_{c}\left(C_{w}-C_{\infty}\right) / \operatorname{Re}_{x} v$ is the concentration buoyancy parameter, $d=v L / u_{0} k$ is the porosity parameter, $\mathrm{Nb}=\tau D_{B\left(C_{w}-C_{\infty}\right)} / v$ is the Brownian motion parameter, $\mathrm{Nb}=\tau D_{T\left(T_{w}-T_{\infty}\right)} / \nu T_{\infty}$ is the thermophoresis parameter, $S=Q L / \rho c u_{0}$ is the heat source/sink parameter, $\operatorname{Pr}=\mu c_{p} / k$ is the Prandtl number, $\mathrm{Sc}=\nu / D_{B}$ is the Schmidt number, $\sigma=k_{r}^{2} / c$ is the ratio rate parameter, $E=E_{a} / k_{1} T_{\infty}$ is the activation energy parameter, and $\zeta=$ $T_{w}-T_{\infty} / T_{\infty}$ is the temperature difference parameter.

Local skin friction coefficient $\left(C_{f}\right)$, local Nusselt number $\left(\mathrm{Nu}_{x}\right)$, and local Sherwood number $\left(\mathrm{Sh}_{x}\right)$ are defined as follows:

$$
\begin{aligned}
C_{f} & =\frac{2 \tau_{w}}{\rho u_{w}^{2}}, \\
\mathrm{Nu}_{x} & =\frac{x p_{w}}{k\left(T_{w}-T_{\infty}\right)}, \\
\mathrm{Sh}_{x} & =\frac{x q_{w}}{D_{B}\left(C_{w}-C_{\infty}\right)},
\end{aligned}
$$


where

$$
\begin{aligned}
& \tau_{w}=\mu\left(\frac{\partial u}{\partial r}+\frac{\Gamma}{\sqrt{2}}\left(\frac{\partial u}{\partial r}\right)^{2}\right)_{r=R}, \\
& p_{w}=-k\left(\frac{\partial T}{\partial r}\right)_{r=R}, \\
& q_{w}=-D_{B}\left(\frac{\partial C}{\partial r}\right)_{r=R} .
\end{aligned}
$$

The nondimensional forms of the local Nusselt number, local skin friction coefficient, and local Sherwood number are

$$
\begin{aligned}
C_{f} \frac{\sqrt{\mathrm{Re}_{x}}}{2} & =f^{\prime \prime}(0)+\frac{\lambda}{2} f^{\prime \prime 2}(0), \\
\mathrm{Nu}_{x} \operatorname{Re}_{x}^{-(1 / 2)} & =-\theta(0), \\
\mathrm{Sh}_{x} \mathrm{Re}_{x}^{-1 / 2} & =-\phi(0),
\end{aligned}
$$

where $\operatorname{Re}_{x}=u_{0}^{2} x / v$ is the local Reynolds number.

\section{Solution Methodology}

The coupled nonlinear ordinary differential equations (7)-(9) subjected to the boundary conditions (10) and (14) are solved numerically using the Runge-Kutta method with shooting technique. For the minor change of initial guesses $u_{1}$, $\bigotimes u_{2}$, and $u_{3}$, the Newton-Raphson method is applied sub- ject to the tolerance $\varepsilon=10^{-5}$. On the origin of a number of computational experiments, there is no important change in the results after $\eta=5$, so we are considering $[0,5]$ as the domain of the problem in terms of $[0, \infty)$. To solve this problem by using this method, equations (7)-(9) are converted to a system of first order ordinary differential equations. The system of first order ordinary differential equations is defined:

$$
\begin{gathered}
f=y_{1}, \\
f^{\prime}=y_{2}, \\
f^{\prime \prime}=y_{3}, \\
f^{\prime \prime \prime}=y^{\prime}, \\
\theta=y_{4}, \\
\theta^{\prime}=y_{5}, \\
\theta^{\prime \prime}=y_{5}^{\prime}, \\
\phi=y_{6}, \\
\phi^{\prime}=y_{7}, \\
\phi^{\prime \prime}=y_{7}^{\prime} .
\end{gathered}
$$

Thus, the systems of first order concurrent ODEs are

$$
\begin{aligned}
& y_{1}^{\prime}=y_{2}, \\
& y_{2}^{\prime}=y_{3}, \\
& y_{3}^{\prime}=\frac{-2 A y_{3}-(3 / 2)(1+2 \eta A)^{1 / 2} A \lambda y_{3}^{2}-y_{1} y_{3}+y_{2}^{2}+(d+M) y_{2}-P y_{4}-q y_{6}}{(1+2 \eta A)+\lambda(1+2 \eta A)^{3 / 2} y_{3}}, \\
& y_{4}^{\prime}=y_{5}, \\
& y_{5}^{\prime}=\frac{-\operatorname{PrNb}(1+2 \eta A) y_{5} y_{7}-2 A y_{5}-\operatorname{Pr} y_{1} y_{5}-\operatorname{PrNt}(1+2 \eta A) y_{5}^{2}+\operatorname{Pr} S_{4}+\operatorname{Pr} \beta y_{2} y_{4}}{1+2 \eta A}, \\
& y_{6}^{\prime}=y_{7}, \\
& y_{7}^{\prime}=\frac{-S c y_{1} y_{7}+\operatorname{PrNt}(1+2 \eta A) y_{5} y_{7}+2 A y_{7}+4(\mathrm{Nt} / \mathrm{Nb}) A y_{5}-2(\mathrm{Nt} / \mathrm{Nb}) \operatorname{Pr} y_{1} y_{5}+2 \operatorname{Pr}\left(\mathrm{Nt}{ }^{2} / \mathrm{Nb}\right)(1+2 \eta A) y_{5}^{2}+2(\mathrm{Nt} / \mathrm{Nb}) \operatorname{Pr} \operatorname{Py}_{4}-2(\mathrm{Nt} / \mathrm{Nb}) \operatorname{Pr} \beta y_{2} y_{4}+\operatorname{Sc\sigma }\left(1+\zeta y_{4}\right)^{e} \exp \left(-E /\left(1+\zeta y_{4}\right)\right) y_{6}+\operatorname{Scc} y_{2} y_{6}}{1+2 \eta A} .
\end{aligned}
$$

Here, the prime represents the derivative with respect to $\eta$, and the transformed boundary conditions are

$$
\begin{aligned}
& y_{1}(0)=\delta, \\
& y_{2}(0)=1+\gamma u_{1}, \\
& y_{3}(0)=u_{1}, \\
& y_{4}(0)=1,
\end{aligned}
$$

$$
\begin{gathered}
y_{5}(0)=u_{2}, \\
y_{6}=1, \\
y_{7}=u_{3} .
\end{gathered}
$$

\section{Result and Discussion}

Figures 2(a)-2(c) are plotted to show the distribution of velocity, temperature, and concentration for different values of the suction $(\delta)$ parameter, respectively. From these figures, 


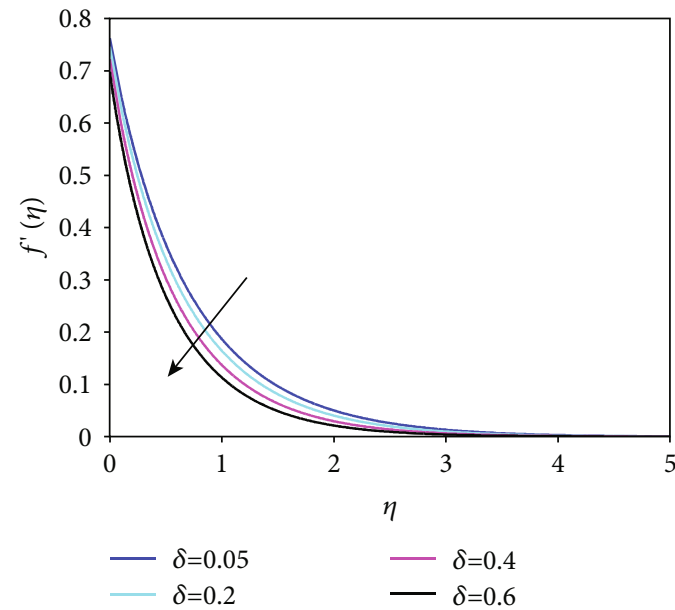

(a)

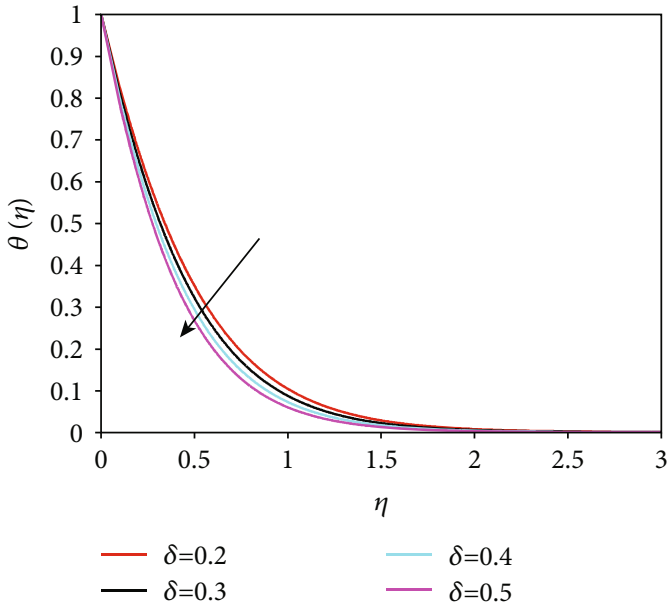

(b)

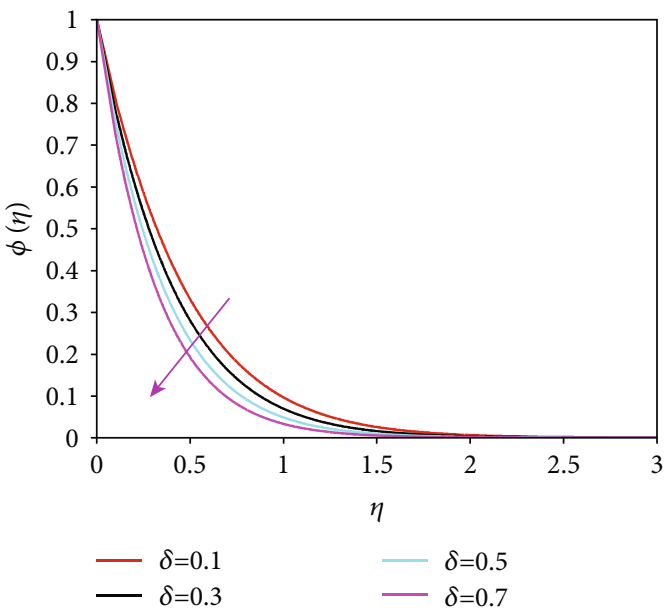

(c)

Figure 2: The graph of velocity, temperature, and concentration for different values of suction $(\delta)$.

it can be seen that when the values of the suction parameter increases, the velocity, temperature, and concentration boundary layer thickness are reduced. This is due to the fact that suction or blowing is the method of controlling the boundary layer. The effect of suction consists in the removal of decelerated fluid particles from the boundary layer before they are given a chance to cause separation. Figures 3(a) and 3 (b) reveal the profiles of velocity and temperature for different values of injection $(-\delta)$. The graph shows that with an increase of the injection parameter, velocity and temperature upsurge.

For different values of slip parameter $(\gamma)$, the variation of velocity and temperature is plotted in Figures 4(a) and 4(b). Figure 4(a) shows that when the value of the slip parameter raises, the velocity profiles reduce. This is because of the fact that when the slip parameter increases, slip velocity increases, and consequently, fluid velocity declines because under the slip condition, the pulling of the stretching wall can only be partly conveyed to the fluid. Figure 4(b) displayed that the temperature profiles raise with an increase of the slip parameter. Figures 5(a), 5(b), and 5(c) are plotted to show the veloc- ity, temperature, and concentration profiles, respectively, for various values of the magnetic field. It is noticed in Figure 5(a).

The graph of velocity, temperature, and concentration is plotted in Figures 6(a), 6(b), and 6(c), respectively, for different values of the porosity parameter. The porous medium causes higher restriction to the fluid flow, which in turn decelerates its motion. Consequently, from Figure 6(a), we observed that with an increase of the porosity parameter, the velocity profiles decrease. But from Figures 6(b) and 6(c), it can be seen that with an increase of the permeability parameter, both temperature and concentration profiles are increased. The distribution of velocity and temperature for different values of curvature parameter $(A)$ is plotted in Figures $7(\mathrm{a})$ and $7(\mathrm{~b})$, respectively. Figure 7 (a) displays that the velocity profiles are increased with an increase of the curvature parameter whereas Figure 7(b) speculates that when the values of the curvature parameter upsurge, the temperature boundary layer thickness declines. This is due to the fact that when the curvature parameter increases, the radius of the cylinder 


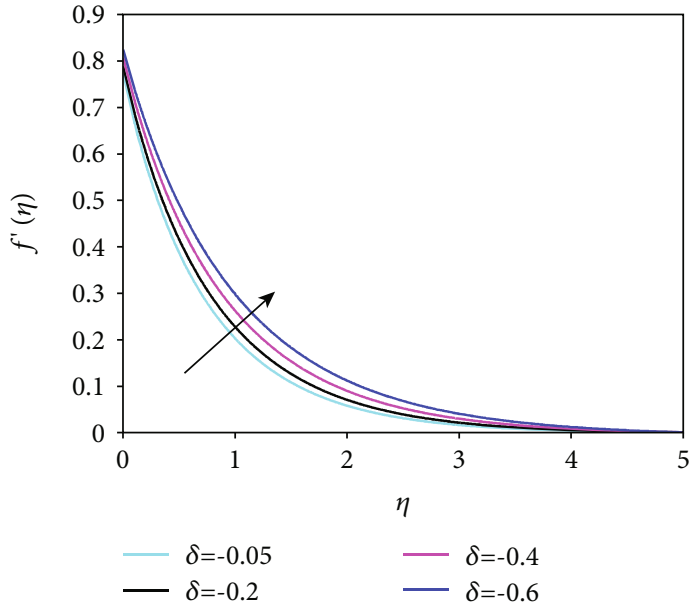

(a)

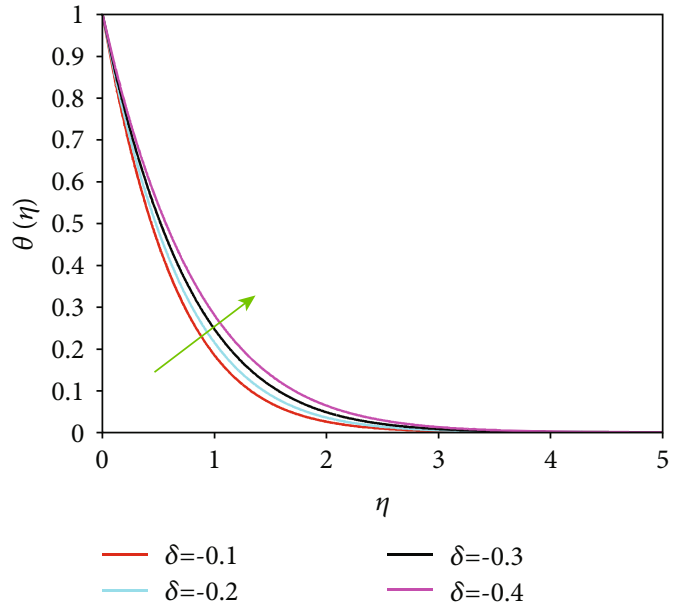

(b)

FIGURE 3: The graph of velocity and temperature for different values of injection $(-\delta)$.

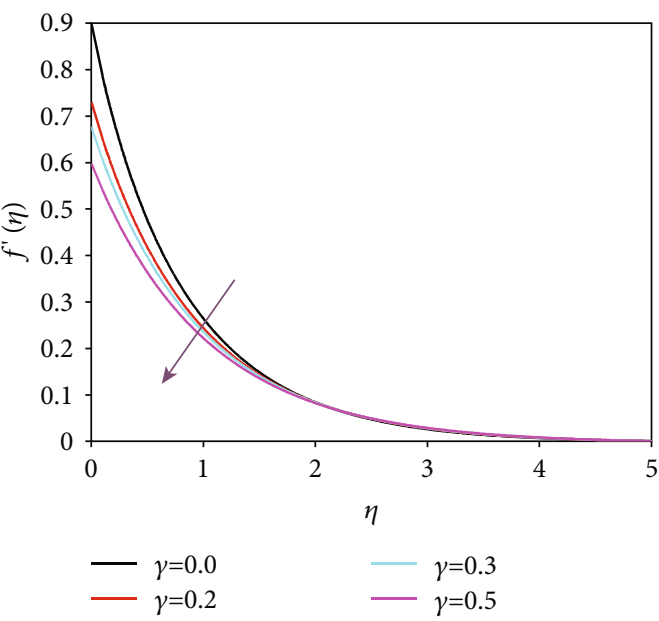

(a)

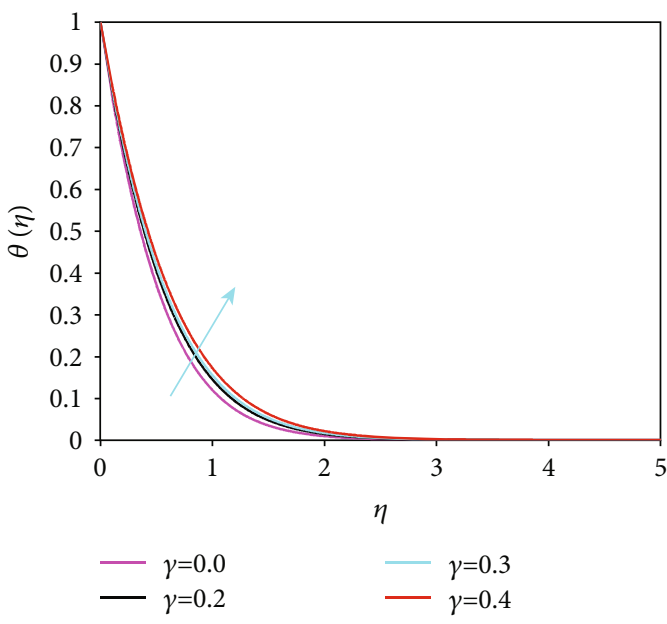

(b)

Figure 4: The graph of velocity and temperature for different values of slip parameter $\gamma$.

reduces which deduces that the contact area of the cylinder with the fluid reduces. Hence, less resistance is offered by the surface to the fluid motion, and as a result, the fluid velocity improved.

For different values of temperature buoyancy parameter $(p)$, the graph of velocity, temperature, and concentration is presented in Figures 8(a)-8(c). From these figures, we observed that velocity profiles increase with an increase of the temperature buoyancy parameter, but temperature and concentration field decrease with an increase of the temperature buoyancy parameter. Figures 9(a)-9(c) show the graph of temperature for different values of heat source/sink parameter $(S)$ and temperature exponent $(\beta)$. It is seen that the temperature field increases with an increase of the heat source, but an opposite trend is happening for large values of heat sink and temperature exponent $(\beta)$. Figures 10(a) and 10 (b) display the graph of velocity, temperature, and concentration, respectively, for various values of concentration buoyancy parameter $(q)$. The figures illustrate that the velocity field increases whereas temperature and concentration fields decline with an increase of the concentration buoyancy parameter.

The effect of activation energy $(E)$ and reaction rate parameter $(\sigma)$ on the concentration field is plotted in Figures 11(a) and 11(b). From these figures, it is seen that when activation energy upsurges, the concentration field rises, but it diminishes with an increase of the reaction rate parameter. This is due to the fact that large activation energy and low temperature cause a smaller reaction rate constant and thus slow down the chemical reaction. So, the concentration of the solute increases. Figures 12(a), 12(b), and 12(c) execute the impact of the Williamson parameter on velocity, temperature, and concentration fields, respectively. Figure 12(a) tells us that with an increase of the Williamson 

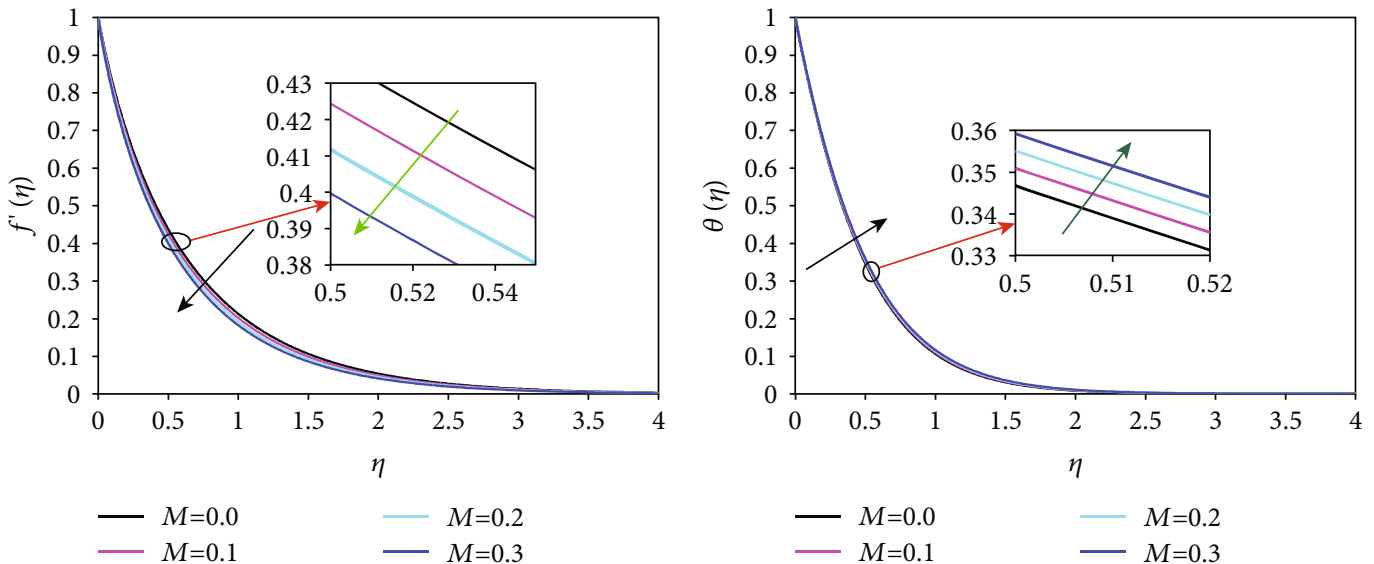

(a)

(b)

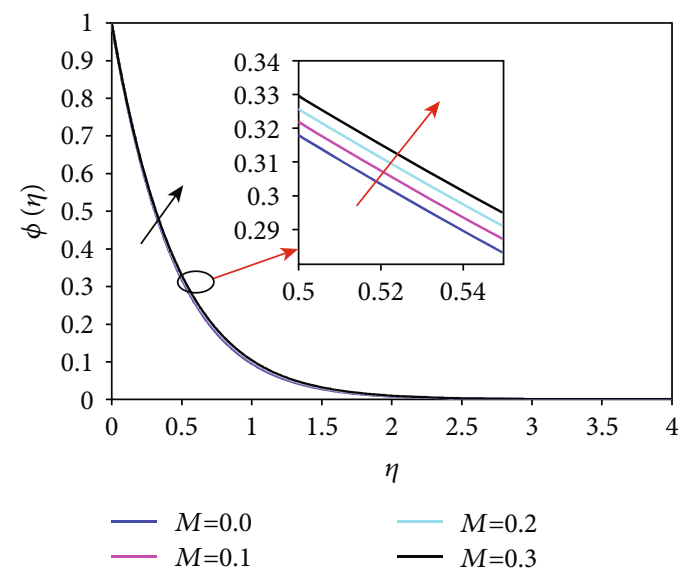

(c)

FiguRE 5: The graph of velocity, temperature, and concentration for different values of magnetic field $(M)$.

parameter, the velocity profiles decrease. As we know that the Williamson parameter is the ratio of relaxation time to retardation time, hence, as the values of the retardation time decrease, the Williamson parameter increases which displays that there is a reduction in the velocity field, and in addition, boundary layer thickness decreases. On behalf of Figures 12(b) and 12(c), it is noted that the temperature and concentration fields upsurge with an enhancement of the Williamson parameter.

The variation of concentration profiles for various values of fitted rate constant $(e)$ and concentration exponent $(\varsigma)$ is shown in Figures 13(a) and 13(b). It is noticed that the distribution of concentration profiles is decreased with an increase in both fitted rate constant and concentration exponent parameters. This is because when the values of the fitted rate constant increased, the factor $\sigma(1+\zeta \theta)^{e} \exp ((-E) /(1+\zeta \theta))$ is augmented. This finally favors the destructive chemical reaction which causes concentration gradient rise. So, the diminution in the concentration field is accompanied by a higher concentration gradient at the wall. The impact of Brownian motion $(\mathrm{Nb})$, thermophoresis parameter $(\mathrm{Nt})$, and Prandtl number ( $\mathrm{Pr}$ ) on temperature is plotted in Figures 14(a)-14(c). Figure 14(a) indicates that when the
Brownian motion $(\mathrm{Nb})$ parameter increases, the movement of nanoparticles from the hot surface to the cold surface happens and ambient fluid occurred. Due to this, the temperature and thermal boundary layer thickness rise. From Figure 14(b), it is observed that the temperature field grows with an increase of the thermophoresis parameter. A phenomenon in which small particles are pulled away from the hot surface to the cold one is called thermophoresis. So, when the surface is heated, the large number of nanoparticles is moved away which raises the temperature of the fluid. Therefore, the temperature of fluid increases. Figure 14(c) displays the effect of the Prandtl number $(\mathrm{Pr})$ on temperature. From this, it can be seen that when the values of Pr rise, the temperature profiles decline. This is because fluid with higher Pr has relatively low thermal conductivity, which results in heat conduction and thereby thermal boundary layer thickness and temperature drop. The effect of Brownian motion $(\mathrm{Nb})$, thermophoresis parameter $(\mathrm{Nt})$, Schmidt number $(\mathrm{Sc})$, and temperature difference parameter $(\zeta)$ on concentration profiles is plotted in Figures 15(a)-15(d). Figure 15(a) indicates that when Brownian motion $(\mathrm{Nb})$ upsurges, volume fraction of nanoparticles within the boundary layer upsurges. It is interesting to note that Brownian motion of the 


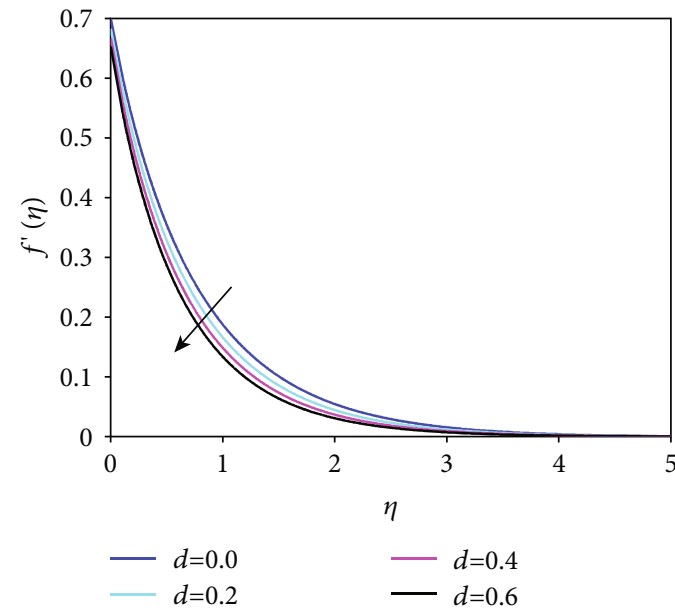

(a)

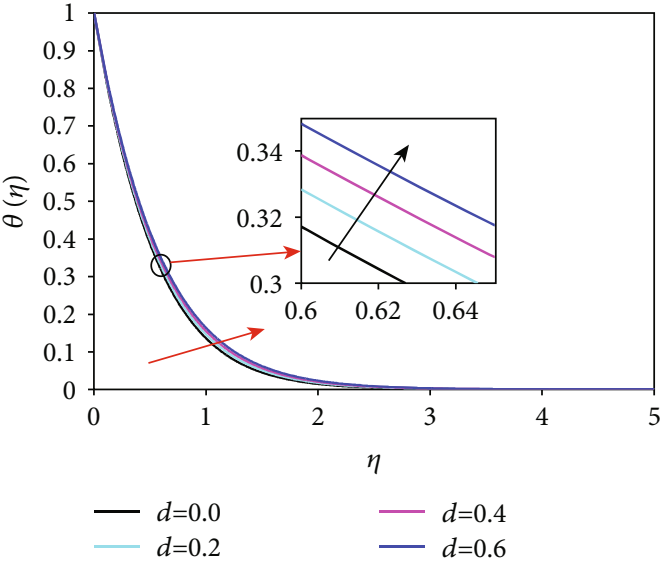

(b)

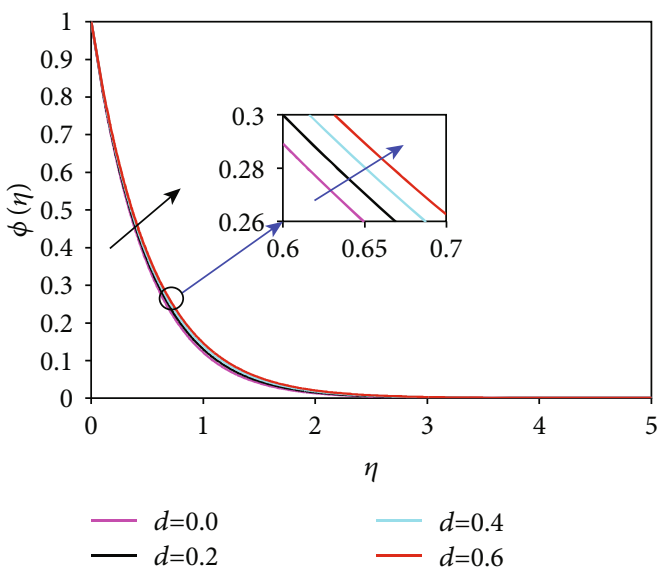

(c)

FIGURE 6: The graph of velocity, temperature, and concentration for different values of porous parameter $(d)$.

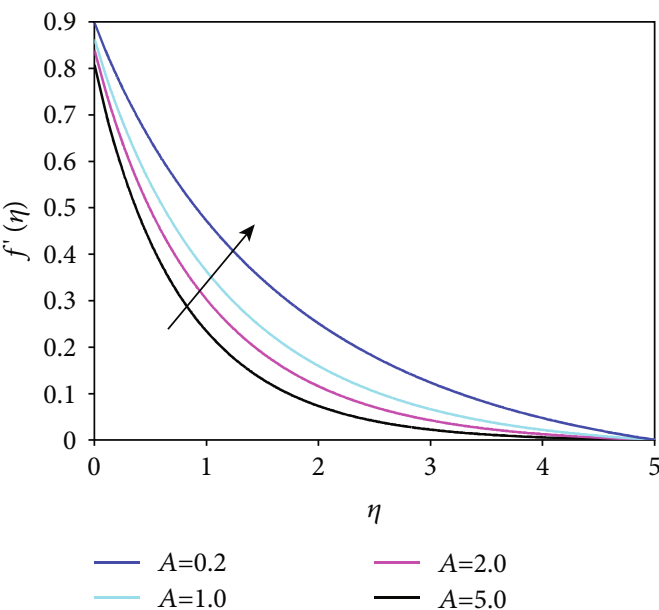

(a)

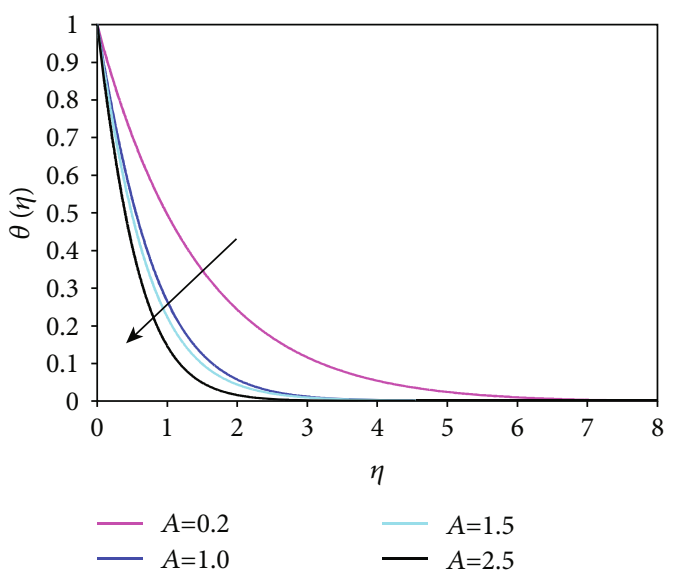

(b)

FIGURE 7: The graph of velocity and temperature for different values of curvature parameter $(A)$.

nanoparticles at the molecular and nanoscale levels is a key mechanism in governing their thermal behavior. In the nanofluid system, due to the size of the nanoparticles, Brow- nian motion affects the heat transfer properties. As the particle size scale approaches to the nanometer scale, Brownian motion on the surrounding liquids plays an important role 


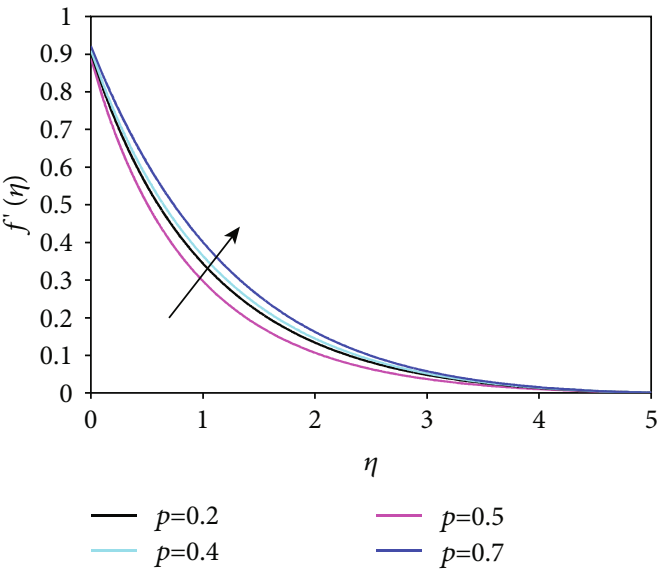

(a)

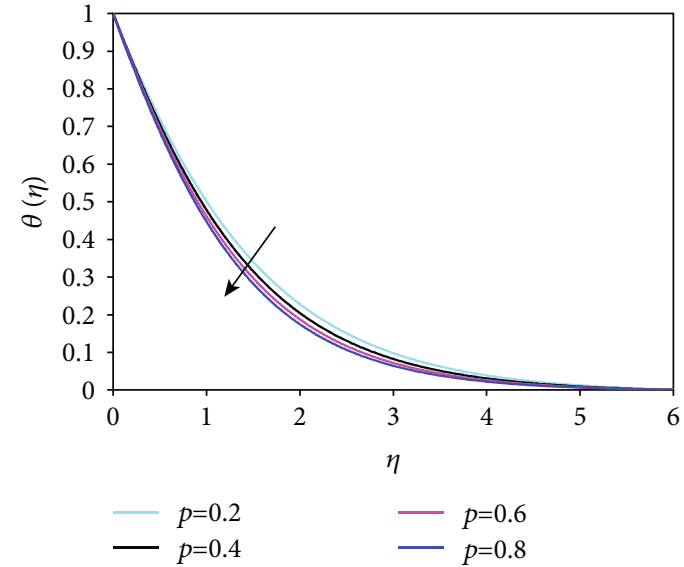

(b)

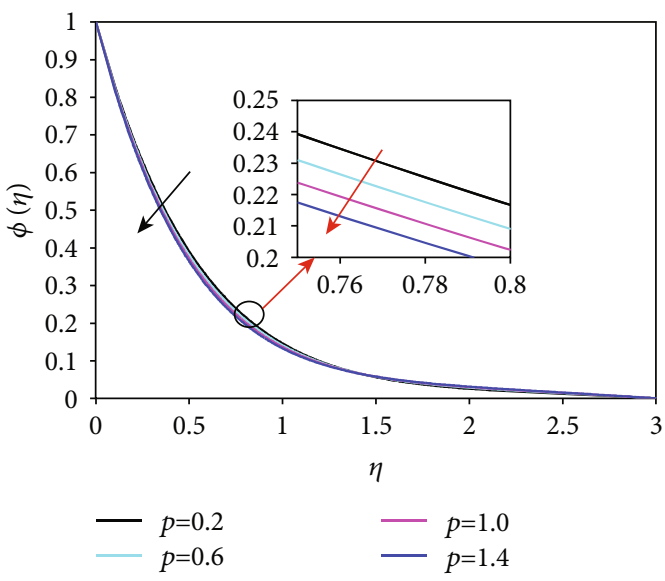

(c)

FiguRE 8: The graph of velocity, temperature, and concentration for different values of temperature buoyancy parameter ( $p$ ).

in heat transfer. Figure 15(b) shows that with an increase of the thermophoresis parameter, concentration profiles rise. From Figure 15(c), it is observed that the concentration profiles decrease with an increase of the Schmidt number. This is due to the fact that weaker molecular diffusivity appeared for a larger Schmidt number and stronger molecular diffusivity corresponds to a lower Schmidt number. Figure 15(d) forecasts that with an increase in the temperature difference parameter, the concentration profiles decline.

Table 1 shows the comparison of the skin friction coefficient with the available published results of Malik et al. [2] and found that it is an excellent agreement. Moreover, the comparisons of the values of the Nusselt number and Sherwood number with the other published result of Hayat et al. [33] are shown in Table 2. Here, from the table, it can be seen that there is an excellent agreement.

Straightforwardly, the numerical values of the skin friction coefficient, Nusselt number, and Sherwood number appear in Table 3 for different values of suction parameter $(\delta)$, permeability parameter $(d)$, curvature parameter $(A)$, temperature buoyancy parameter $(p)$, concentration buoyancy parameter $(q)$, and slip parameter $(\gamma)$. It is noticed that the magnitude of the skin friction coefficient increases with an increase of suction parameter $(\delta)$, permeability parameter $(d)$, and curvature parameter $(A)$ whereas with an upsurge of temperature buoyancy parameter $(p)$, concentration buoyancy parameter $(q)$, and slip parameter $(\gamma)$, the skin friction coefficient magnitude was reduced, but it remains constant with an increase of activation energy $(E)$ and reaction rate parameter $(\sigma)$. Moreover, from this table, it can be seen that when the values of suction parameter $(\delta)$, temperature buoyancy parameter $(p)$, concentration buoyancy parameter $(q)$, activation energy $(E)$, and curvature parameter $(A)$ increase, the magnitude of the Nusselt number increases whereas with an increase of permeability parameter $(d)$, reaction rate parameter $(\sigma)$, and slip parameter $(\gamma)$, there is a magnitude of Nusselt number diminution. Furthermore, from this table, it can be seen that the Sherwood number declines when the values of permeability parameter $(d)$, activation energy $(E)$, and slip parameter $(\gamma)$ rise, but an opposite trend is happening on the Sherwood number as the values of suction parameter $(\delta)$, temperature buoyancy parameter $(p)$, concentration buoyancy parameter $(q)$, and reaction rate parameter $(\sigma)$. 


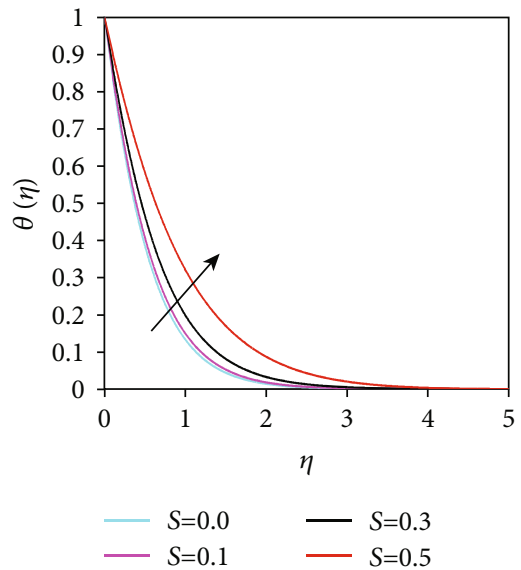

(a)

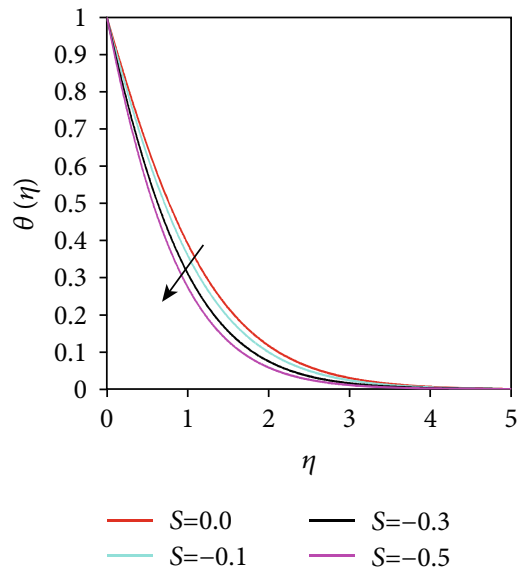

(b)

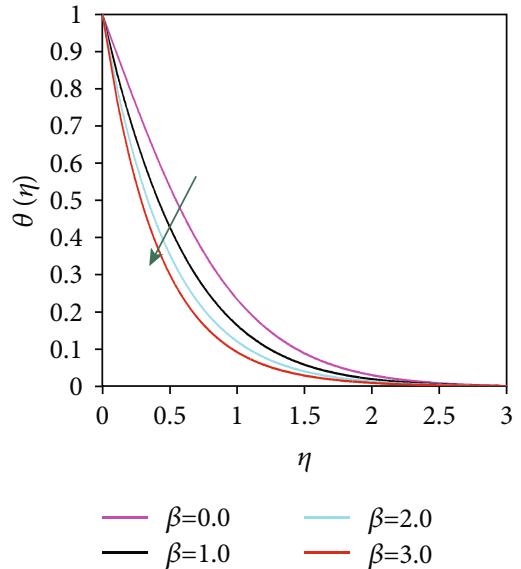

(c)

FIGURE 9: The graph of temperature for different values of heat source/sink parameter $(S)$ and temperature exponent $\beta$.

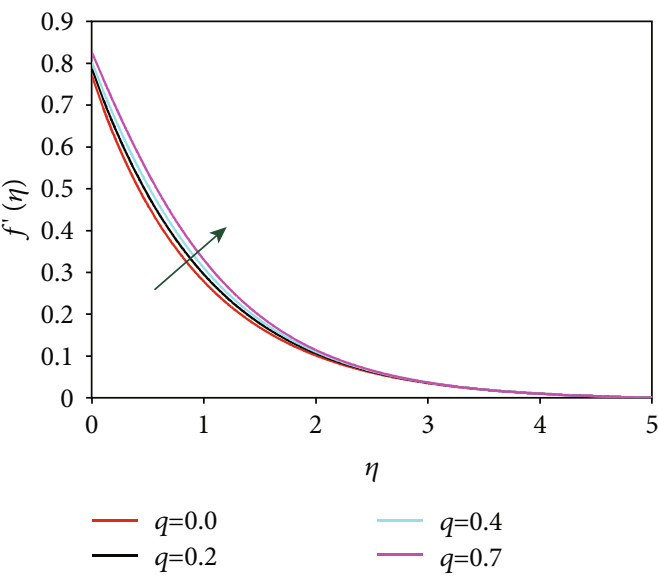

(a)

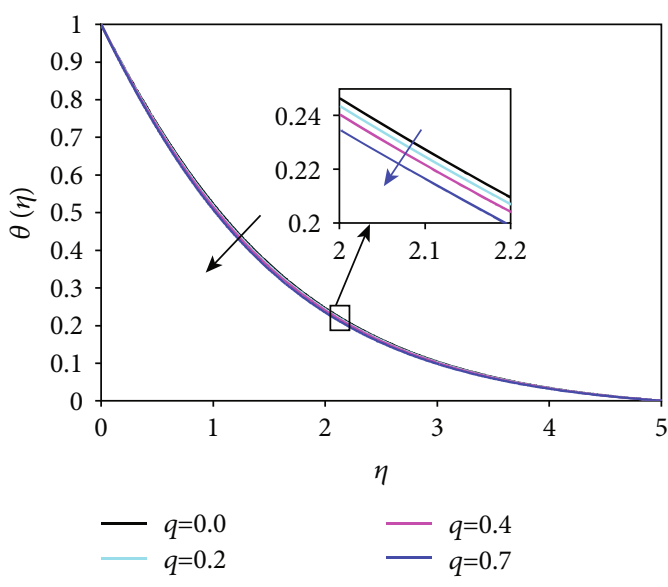

(b)

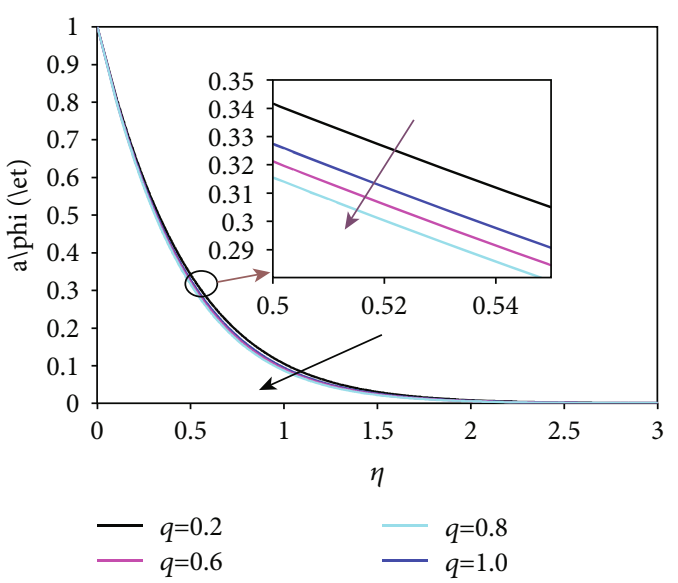

(c)

FIGURE 10: The graph of velocity, temperature, and concentration for different values of concentration buoyancy parameter $(q)$.

\section{Conclusion}

This article deals with numerical solution of MHD nonNewtonian nanofluid over a stretching cylinder embedded in a porous medium with the effect of activation energy and heat generation/absorption. The impact of various dimensionless physical parameters on velocity, temperature, and concentration profiles as well as skin coefficient friction $\left(C_{f}\right)$, heat 


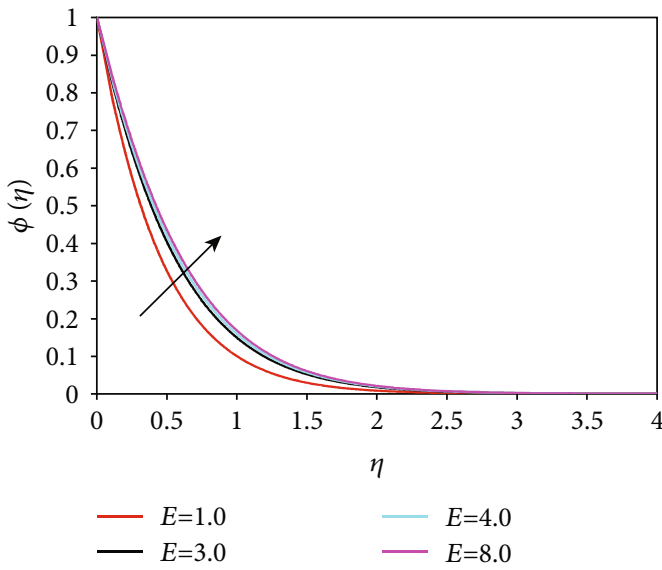

(a)

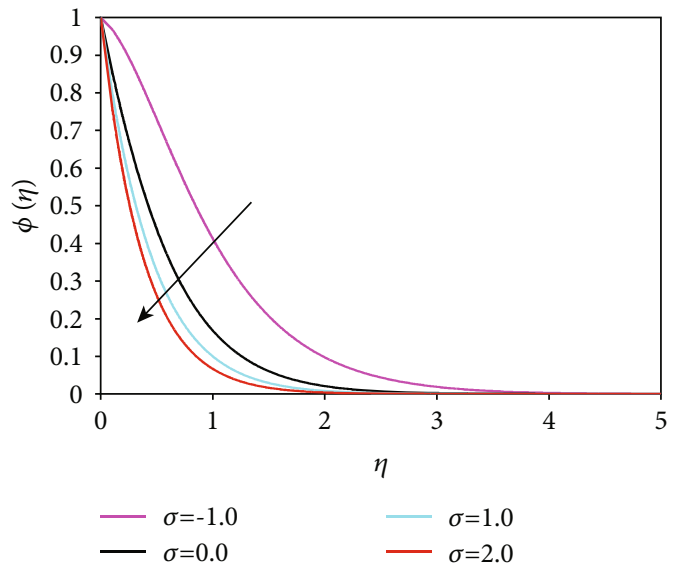

(b)

Figure 11: The graph of concentration for different values of activation energy $(E)$ and reaction rate parameter $\sigma$.

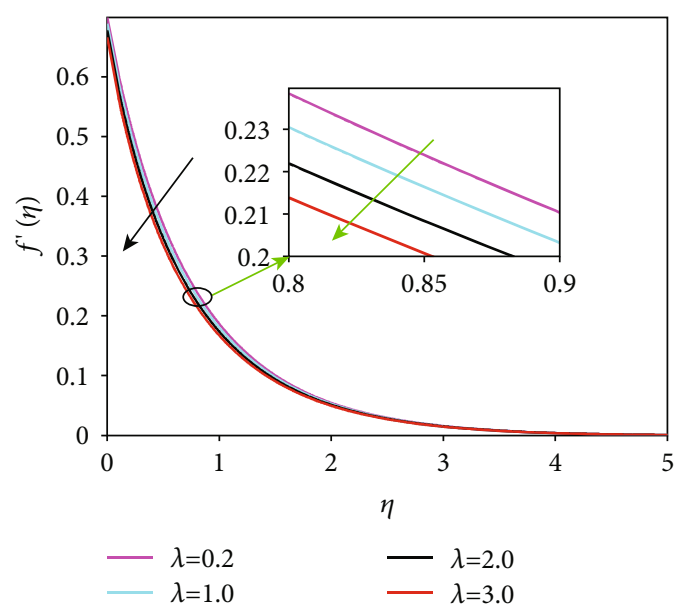

(a)

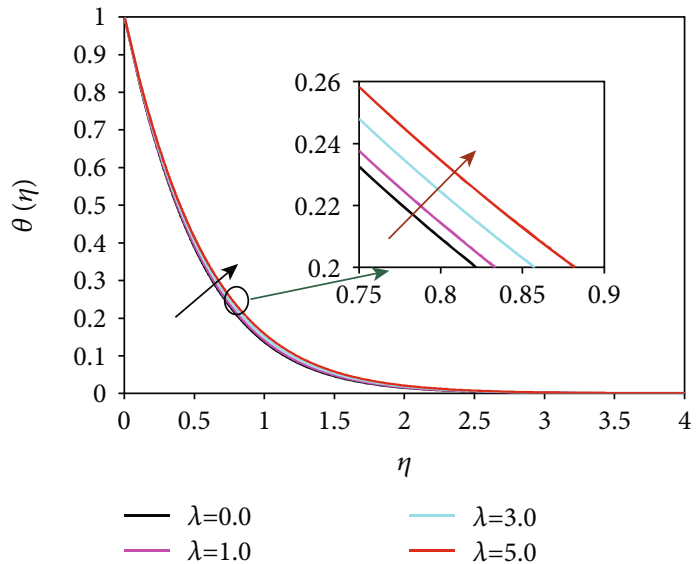

(b)

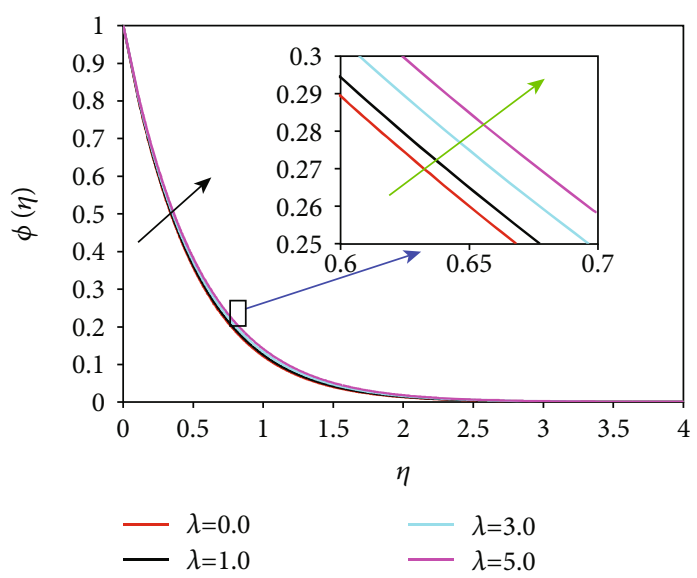

(c)

FIGURE 12: The graph of velocity, temperature, and concentration for different values of Williamson parameter $\lambda$.

transfer rate $\left(\mathrm{Nu}_{x}\right)$, and mass transfer rate parameter $\left(\mathrm{Sh}_{x}\right)$ is discussed in graphs and tables. The summarized conclusion of numerical study is as follows: (i) The suction $(\delta)$ parameter interaction reduces the velocity, temperature, and concentration profiles of the flow 


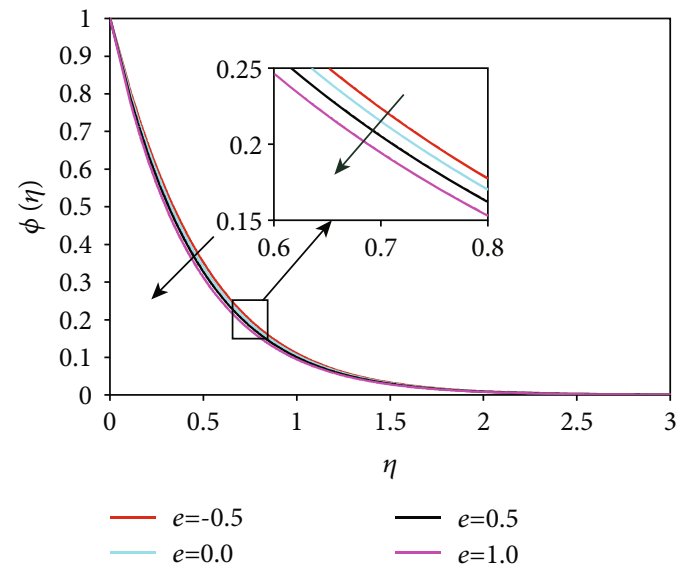

(a)

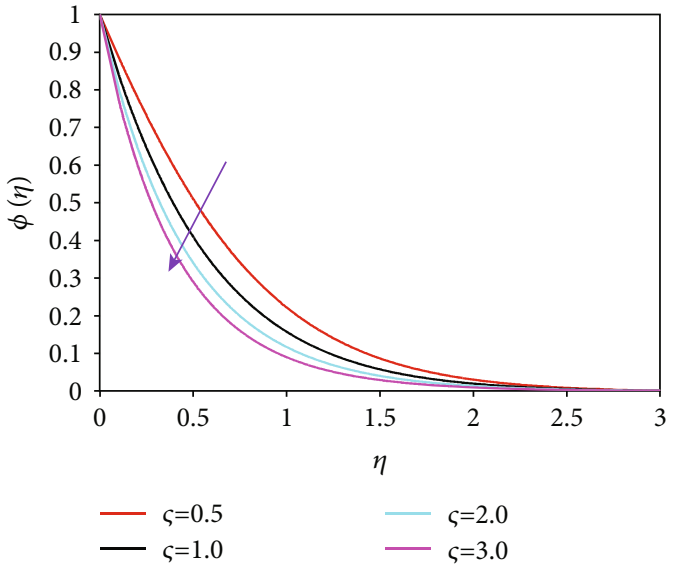

(b)

Figure 13: The graph of concentration for different values of fitted rate constant $(e)$ and concentration exponent $\varsigma$.

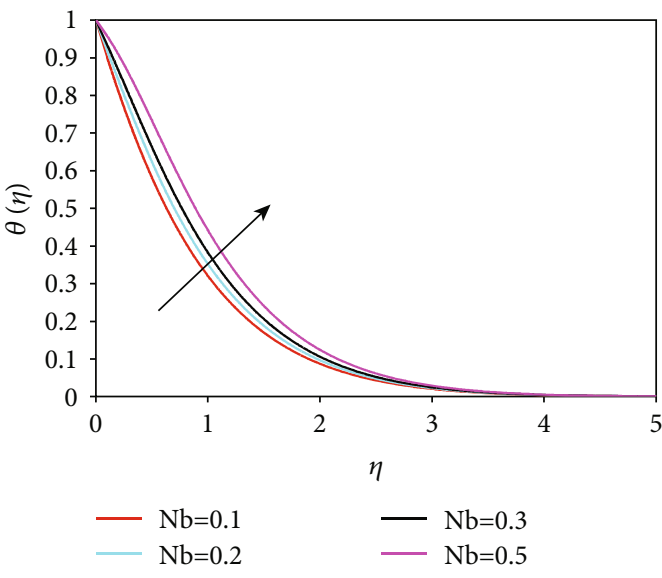

(a)

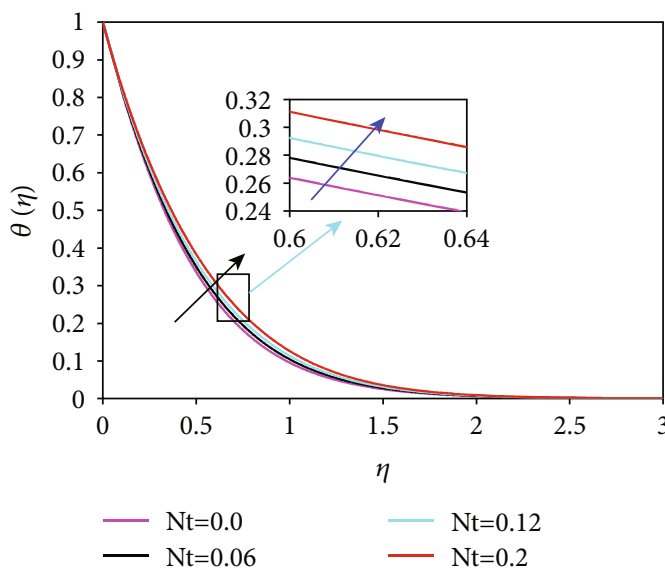

(b)

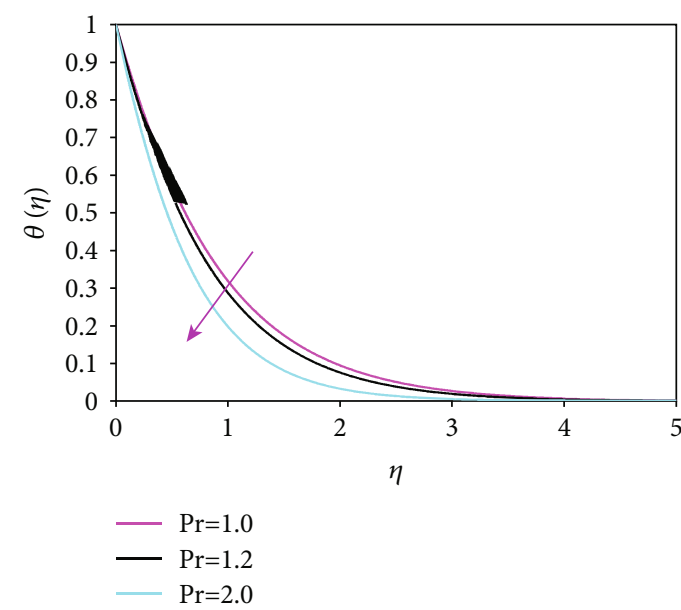

(c)

FiguRE 14: The graph of temperature for different values of Brownian motion ( $\mathrm{Nb})$, thermophoresis parameter (Nt), and Prandtl number (Pr).

(ii) Curvature parameter $(A)$ improves velocity profiles, but it reduces temperature profiles

(iii) With an increase of porous parameter $(d)$, the profiles of velocity decline whereas temperature and concentration profiles are improved (iv) Raise in temperature and concentration buoyancy parameter enhances velocity whereas temperature and concentration are reduced

$(v)$ Increase in activation energy $(E)$ enhances concentration profiles 


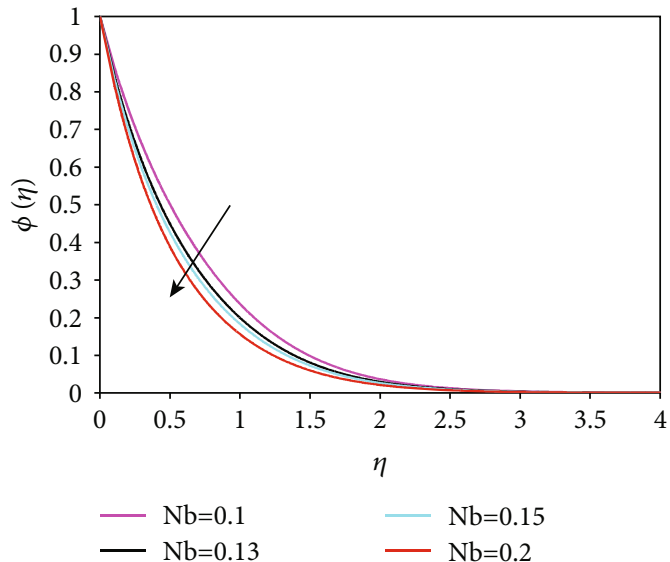

(a)

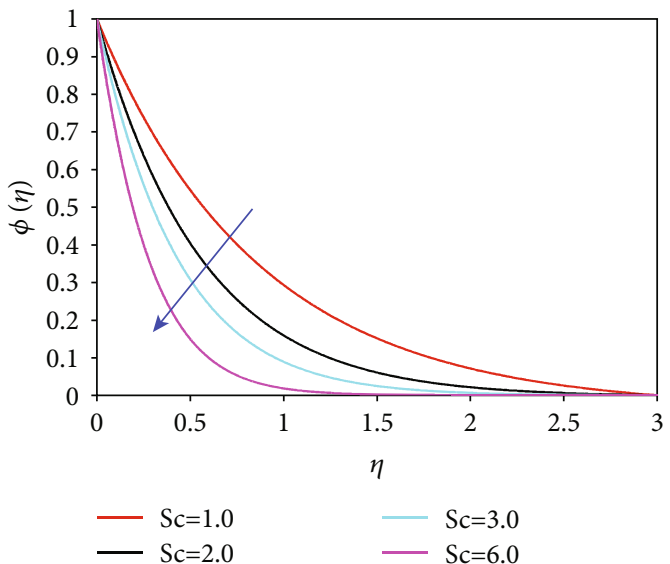

(c)

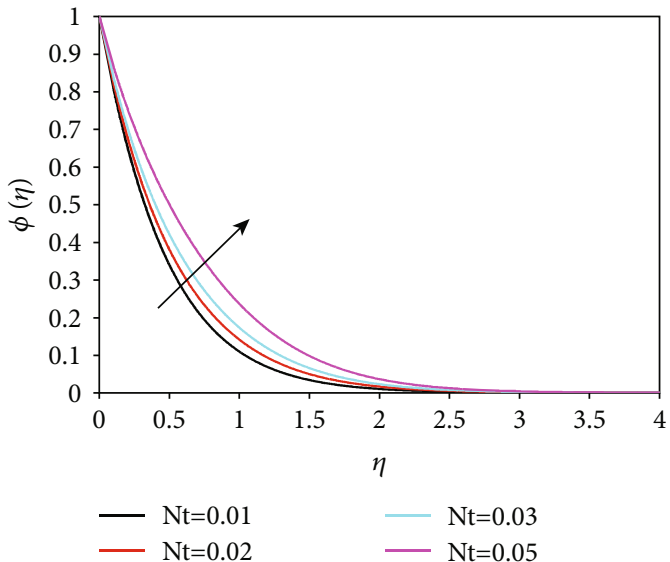

(b)

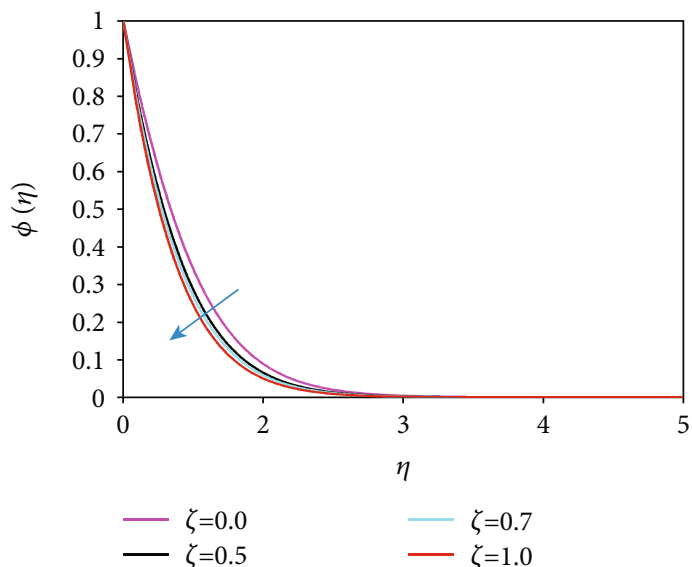

(d)

FIGURE 15: The graph of concentration for different values of Brownian motion (Nb), thermophoresis parameter (Nt), Schmidt number (Sc), and temperature difference parameter $(\zeta)$; the velocity declines with an increase of the magnetic field parameter. From (b, c), it can be seen that the temperature and concentration upsurge with an increase of the magnetic field parameter $(M)$. The physical meaning of this behavior is an upsurge in the values of the magnetic field parameter develops the force opposite to the flow, which is known as Lorentz force. This body force reduced the boundary layer flow and congeals the velocity boundary layers. Furthermore, this force creates a resistance force that opposes the fluid motion. Therefore, for the higher magnetic field, heat is generated leading to a boost in the temperature and thermal boundary layer thickness.

TABLE 1: Comparison of values of skin friction coefficient $\left(f^{\prime \prime}(0)\right)$ for various values of $\lambda$ with earlier scholar results.

\begin{tabular}{ccc}
\hline$\Lambda$ & $\begin{array}{c}\text { Malik et al. [2] } \\
f^{\prime \prime}(0)\end{array}$ & $\begin{array}{c}\text { Present results } \\
f^{\prime \prime}(0)\end{array}$ \\
\hline 0.1 & -0.9776 & -0.9779 \\
0.2 & -0.9118 & -0.9128 \\
0.3 & -0.8413 & -0.8417 \\
\hline
\end{tabular}

(vi) With an increase of reaction rate parameter $(\sigma)$, the magnitude of the skin friction coefficient remains constant but the magnitude of Nusselt number diminution and Sherwood number rises

(vii) Sherwood number declines when the value of slip parameter $(\gamma)$ rises.
TABLE 2: Comparison of values of the Nusselt number $\left(-\theta^{\prime}(0)\right)$ and Sherwood number $\left(-\phi^{\prime}(0)\right)$ for various values of $\operatorname{Pr}$ and $E$ with earlier scholar results.

\begin{tabular}{llcccc}
\hline Pr & $E \begin{array}{c}\text { Hayat et al. [33] } \\
\left(-\theta^{\prime}(0)\right)\end{array}$ & $\begin{array}{c}\text { Present } \\
\text { result }\end{array}$ & $\begin{array}{c}\text { Hayat et al. [33] } \\
\left(-\phi^{\prime}(0)\right)\end{array}$ & $\begin{array}{c}\text { Present } \\
\text { result }\end{array}$ \\
\hline 1 & 1 & 0.51903 & 0.51913 & & \\
2 & & 0.85109 & 0.85121 & & \\
5 & & 1.51553 & 1.51558 & & \\
7 & & 1.84540 & 1.84547 & & \\
10 & & 2.26033 & 2.26039 & & 0.95069 \\
& 1 & & & 0.95064 & 0.75338 \\
& 2 & & & 0.75333 & 0.58020 \\
& 4 & & & 0.58015 & 0.53530 \\
& 6 & & & 0.53528 & 0.52371 \\
\hline
\end{tabular}


TABle 3: Numerical results of the skin friction coefficient, Nusselt number, and Sherwood number for different values of $\delta, d, p$, $q, \gamma, E, R$, and $\sigma$ when $\mathrm{Nt}=0.1, \mathrm{Nb}=0.1, M=0.3, \lambda=0.1, e=0.1$, $\varsigma=1.0, \zeta=0.1, \mathrm{Sc}=3, \operatorname{Pr}=2, \beta=1$, and $S=0.1$.

\begin{tabular}{|c|c|c|c|c|c|c|c|c|c|c|}
\hline$\delta$ & $d$ & $p$ & $Q$ & $E$ & $A$ & $\sigma$ & $\gamma$ & $-f^{\prime \prime}(0)$ & $-\theta^{\prime}(0)$ & $-\phi^{\prime}(0)$ \\
\hline 0.0 & 0.0 & 0.1 & 0.1 & 1.0 & 0.0 & 0.1 & 0.0 & 1.5944 & 1.3886 & 1.8893 \\
\hline 0.1 & & & & & & & & 1.7116 & 1.4823 & 2.0540 \\
\hline 0.2 & & & & & & & & 1.8482 & 1.5808 & 2.2277 \\
\hline 0.3 & & & & & & & & 2.0143 & 1.6829 & 2.4095 \\
\hline & 0.0 & & & & & & & 1.6684 & 1.6015 & 2.2530 \\
\hline & 0.2 & & & & & & & 1.8482 & 1.5805 & 2.2277 \\
\hline & 0.4 & & & & & & & 2.0500 & 1.5602 & 2.2029 \\
\hline & 0.6 & & & & & & & 2.3045 & 1.5402 & 2.1784 \\
\hline & & 0.1 & & & & & & 1.7628 & 1.5895 & 2.2387 \\
\hline & & 0.3 & & & & & & 1.6121 & 1.6060 & 2.2588 \\
\hline & & 0.5 & & & & & & 1.4802 & 1.6209 & 2.2771 \\
\hline & & 1.0 & & & & & & 1.2026 & 1.6534 & 2.3467 \\
\hline & & & 0.1 & & & & & 1.6992 & 1.5914 & 2.2461 \\
\hline & & & 0.3 & & & & & 1.5826 & 1.6066 & 2.2600 \\
\hline & & & 0.5 & & & & & 1.4770 & 1.6171 & 2.2731 \\
\hline & & & 1.5 & & & & & 1.2469 & 1.6407 & 2.3024 \\
\hline & & & & 1.0 & & & & 1.8482 & 1.5509 & 1.1909 \\
\hline & & & & 2.0 & & & & 1.8482 & 1.5534 & 1.1781 \\
\hline & & & & 4.0 & & & & 1.8482 & 1.5538 & 1.1711 \\
\hline & & & & 8.0 & & & & 1.8482 & 1.5539 & 1.1699 \\
\hline & & & & & 0.0 & & & 1.5491 & 1.5273 & 2.1991 \\
\hline & & & & & 0.15 & & & 1.8482 & 1.5805 & 2.2277 \\
\hline & & & & & 0.2 & & & 1.9930 & 1.5967 & 2.2352 \\
\hline & & & & & 0.25 & & & 2.2087 & 1.6117 & 2.2413 \\
\hline & & & & & & 0.1 & & 1.8482 & 1.5527 & 1.1909 \\
\hline & & & & & & 0.3 & & 1.8482 & 1.5504 & 1.2315 \\
\hline & & & & & & 0.7 & & 1.8482 & 1.5463 & 1.3080 \\
\hline & & & & & & 1.5 & & 1.8482 & 1.5393 & 1.4457 \\
\hline & & & & & & & 0.0 & 1.8482 & 1.5805 & 2.2277 \\
\hline & & & & & & & 0.2 & 1.1506 & 1.4413 & 2.0311 \\
\hline & & & & & & & 0.3 & 0.9958 & 1.3940 & 1.9643 \\
\hline & & & & & & & 0.4 & 0.8832 & 1.3544 & 1.9083 \\
\hline
\end{tabular}

\section{Nomenclature}

A: $\quad$ Curvature parameter

c: $\quad$ Volumetric volume expansion coefficient

$C_{f}: \quad$ Skin friction coefficient

$d$ : Porosity parameter

$D_{B}: \quad$ Brownian diffusion coefficient

$D_{T}$ : Thermophoretic diffusion coefficient

$e$ : $\quad$ Fitted rate parameter

E: Activation energy

$f$ : Dimensionless stream function

M: $\quad$ Magnetic field

$\mathrm{Nb}$ : Brownian motion parameter

Nt: Thermophoresis parameter

$\mathrm{Nu}_{x}$ : Nusselt number p: $\quad$ Temperature buoyancy parameter

Pr: Prandtl number

$p_{w}: \quad$ Heat flux of the nanofluid

$q$ : $\quad$ Concentration buoyancy parameter

$q_{w}$ : Mass flux of the nanofluid

Re: Reynolds number

$S$ : $\quad$ Heat source/sink parameter

Sc: $\quad$ Schmidt number

$\mathrm{Sh}_{x}$ : Sherwood number

$T: \quad$ Temperature

$T_{\infty}$ : Free stream temperature

$U_{e}: \quad$ Free stream velocity

$(u, v)$ : Velocity components in $x$ and $y$ coordinates

$\psi$ : $\quad$ Stream function

$\theta: \quad$ Dimensionless temperature

$\eta$ : $\quad$ Similarity variable

$\mu$ : $\quad$ Dynamic viscosity

$\beta$ : $\quad$ Temperature exponent parameter

$\alpha: \quad$ Thermal diffusivity of the nanofluid

$\phi$ : Dimensionless concentration function

$(\rho c)_{f}$ : Heat capacity of the fluid

$(\rho c)_{p}$ : Heat capacity of the nanoparticles

$\rho_{p}: \quad$ Density of nanoparticles

$\tau: \quad$ The parameter defined by $(\rho c)_{p} /(\rho c)_{f}$

$\gamma$ : $\quad$ Stretching parameter

$\iota: \quad$ Slip coefficient

$\delta: \quad$ Suction/injection parameter

$\lambda$ : Williamson parameter

$\zeta$ : $\quad$ Temperature difference

$\Gamma$ : $\quad$ Positive time constant

$\sigma: \quad$ Reaction rate parameter

$\sigma_{1}: \quad$ Electrical conductivity of the fluid

$\varsigma$ : Concentration exponent

$\tau_{w}: \quad$ Skin friction or shear stress

$\rho: \quad$ Density

$v$ : Kinematic viscosity.

\section{Data Availability}

Data sharing is not applicable to this paper as no database was generated or analyzed during the contact study.

\section{Conflicts of Interest}

Both authors have no competing interests.

\section{References}

[1] R. V. Williamson, "The flow of pseudoplastic materials," Industrial and Engineering Chemistry, vol. 21, no. 11, pp. 1108-1111, 1929.

[2] M. Y. Malik, M. Bibi, F. Khan, and T. Salahuddin, "Numerical solution of Williamson fluid flow past a stretching cylinder and heat transfer with variable thermal conductivity and heat generation/absorption," AIP Advances, vol. 6, no. 3, article 035101, 2016.

[3] M. R. Krishnamurthy, B. C. Prasannakumara, B. J. Gireesha, and R. S. R. Gorla, "Effect of chemical reaction on MHD boundary layer flow and melting heat transfer of Williamson 
nanofluid in porous medium," Engineering Science and Technology, an International Journal, vol. 19, no. 1, pp. 53-61, 2016.

[4] S. Nadeem, S. T. Hussain, and C. Lee, "Flow of a Williamson fluid over a stretching sheet," Brazilian Journal of Chemical Engineering, vol. 30, no. 3, pp. 619-625, 2013.

[5] M. J. Babu and N. Sandeep, "MHD non-Newtonian fluid flow over a slendering stretching sheet in the presence of crossdiffusion effects," Alexandria Engineering Journal, vol. 55, no. 3, pp. 2193-2201, 2016.

[6] M. S. Khan, M. M. Rahman, S. M. Arifuzzaman, P. Biswas, and I. Karim, "Williamson fluid flow behaviour of mhd convective radiative cattaneo-christov heat flux type over a linearly stretched-surface with heat generation and thermal-diffusion," Frontiers in Heat and Mass Transfer, vol. 9, p. 15, 2017.

[7] S. Maripala and N. Kishan, "Unsteady MHD flow and heat transfer of nanofluid over a permeable shrinking sheet with thermal radiation and chemical reaction," American Journal of Engineering Research (AJER), vol. 4, no. 6, pp. 68-79, 2015.

[8] C. RamReddy and R. R. Kairi, "The effect of melting on mixed convection heat and mass transfer in non-Newtonian nanofluid SATURATEED in porous medium," Frontiers in Heat and Mass Transfer, vol. 6, p. 6, 2015.

[9] L. G. Vijaya, B. L. Anand, and R. K. Srinivasa, "MHD mixed convection stagnation point flow of nanofluid through a porous medium over stretching sheet," International Journal of Pure and Applied Mathematics, vol. 118, no. 10, pp. 369389, 2018.

[10] N. Ambreen, A. Rehman, N. Sheikh, S. Iqbal, and M. Zulfiqar, "Boundary-layer flow and heat transfer over a rotating porous disk in a non-Newtonian Williamson nanofluid," Indian Journal of Science and Technology, vol. 12, no. 38, 2019.

[11] K. A. R. M. Aurangzaib, N. F. Mohammad, and S. Shafie, "Unsteady MHD mixed convection flow with heat and mass transfer over a vertical plate in a micropolar fluid-saturated porous medium," Journal of Applied Science and Engineering, vol. 16, no. 2, pp. 141-150, 2013.

[12] R. G. Bal, G. B. Shankar, and M. N. Raja Shekar, "Numerical solution of MHD mixed convective boundary layer flow of a nanofluid through a porous medium due to an exponentially stretching sheet with magnetic field effect," International Journal of Applied Engineering Research, vol. 14, no. 9, pp. 20742083, 2019.

[13] A. Al-Mamun, S. M. Arifuzzaman, S. Reza-E-Rabbi, P. Biswas, and M. Khan, "Computational Modelling on MHD radiative Sisko nanofluids flow through a nonlinearly stretching sheet," International Journal of Heat and Technology, vol. 37, no. 1, pp. 285-295, 2019.

[14] S. E. Ahmed, R. A. Mohamed, A. E. M. Aly, and M. S. Soliman, "Magnetohydrodynamic Maxwell nanofluids flow over a stretching surface through a porous medium," International Journal of Mechanical, Industrial and Aerospace Sciences, vol. 13, no. 6, 2019.

[15] I. Ullah, S. Shafie, and I. Khan, "Heat generation and absorption in MHD flow of Casson fluid past a stretching wedge with viscous dissipation and Newtonian heating," Jurnal Teknologi, vol. 80, no. 3, pp. 77-85, 2018.

[16] Z. Khan, H. U. Rasheed, T. A. Alkanhal, M. Ullah, I. Khan, and I. Tlili, "Effect of magnetic field and heat source on upperconvected-Maxwell fluid in a porous channel," Open Physics, vol. 16, no. 1, pp. 917-928, 2018.
[17] K. Padmavathi and Y. Anitha Kumari, "MHD mixed convection stagnation-point flow of a power - law nano fluid towards a stretching surface in the presence of viscous dissipation and suction or injection," Aryabhatta Journal of Mathematics and Informatics, vol. 9, no. 1, pp. 457-473, 2017.

[18] S. Panuganti and G. R. Machireddy, "Chemical reaction and viscous dissipation effects on radiative MHD boundary layer flow of nanofluids," Aryabhatta Journal of Mathematics and Informatics, vol. 9, no. 1, pp. 405-430, 2017.

[19] M. D. Shamshuddin, T. Thirupathi, and P. V. Satya Narayana, "Micropolar fluid flow induced due to a stretching sheet with heat source/sink and surface heat flux boundary condition effects," Journal of Applied and Computational Mechanics, vol. 5, no. 5, pp. 816-826, 2019.

[20] K. U. Rehman, M. Y. Malik, A. A. Khan, I. Zehra, M. Zahri, and M. Tahir, "Numerical solution of chemically reactive non-Newtonian fluid flow: Dual stratification," The European Physical Journal Plus, vol. 132, no. 12, article 550, 2017.

[21] S. Anuradha and M. Yegammai, "MHD radiative boundary layer flow of nanofluid past a vertical plate with effects of binary chemical reaction and activation energy," Global Journal of Pure and Applied Mathematics, vol. 13, no. 9, pp. 6377-6392, 2017.

[22] C. S. Sravanthi and R. S. R. Gorla, "Effects of heat source/sink and chemical reaction on MHD Maxwell nanofluid flow over a convectively heated exponentially stretching sheet using homotopy analysis method," International Journal of Applied Mechanics and Engineering, vol. 23, no. 1, pp. 137-159, 2018.

[23] M. Suleman, M. Ramzan, S. Ahmad, and D. Lu, "Numerical simulation for homogeneous-heterogeneous reactions and Newtonian heating in the silver-water nanofluid flow past a nonlinear stretched cylinder," Phys. Scr., vol. 94, no. 8, article 085702, 2019.

[24] A. R. Bestman, "Natural convection boundary layer with suction and mass transfer in a porous medium," International Journal of Energy Research, vol. 14, no. 4, pp. 389-396, 1990.

[25] F. G. Awad, S. Motsa, and M. Khumalo, "Heat and mass transfer in unsteady rotating fluid flow with binary chemical reaction and activation energy," PLoS One, vol. 9, no. 9, article e107622, 2014.

[26] M. Dhlamini, P. K. Kameswaran, P. Sibanda, S. Motsa, and H. Mondal, "Activation energy and binary chemical reaction effects in mixed convective nanofluid flow with convective boundary conditions," Journal of Computational Design and Engineering, vol. 6, no. 2, pp. 149-158, 2019.

[27] S. Anuradha and K. Sasikala, "MHD free convective flow of a nanofluid over a permeable shrinking sheet with binary chemical reaction and activation energy," International Journal of Engineering Science Invention, vol. 7, no. 1, pp. 22-30, 2018.

[28] A. Hamid, Hashim, and M. Khan, "Impacts of binary chemical reaction with activation energy on unsteady flow of magnetoWilliamson nanofluid," Journal of Molecular Liquids, vol. 262, pp. 435-442, 2018.

[29] C.-J. Huang, Arrhenius activation energy effects on free convective about a permeable horizontal cylinder in porous medium, Transport in porous media, 2019.

[30] M. Mustafa, J. A. Khan, T. Hayat, and A. Alsaedi, "Buoyancy effects on the MHD nanofluid flow past a vertical surface with chemical reaction and activation energy," International Journal of Heat and Mass Transfer, vol. 108, pp. 1340-1346, 2017. 
[31] A. Zaib, M. M. Rashidi, A. Chamkha, and K. Bhattacharyya, "Numerical solution of second law analysis for MHD Casson nanofluid past a wedge with activation energy and binary chemical reaction," International Journal of Numerical Methods for Heat \& Fluid Flow, vol. 27, no. 12, pp. 28162834, 2017.

[32] M. Monica, J. Sucharitha, and C. H. Kishore, "Effects of exothermic chemical reaction with Arrhenius activation energy, non-uniform heat source/sink on MHD stagnation point flow of a Casson fluid over a nonlinear stretching sheet with variable fluid properties and slip conditions," Journal of the Nigerian Mathematical Society, vol. 36, pp. 163-190, 2017.

[33] T. Hayat, I. Ullah, M. Waqas, and A. Alsaedi, "Attributes of activation energy and exponential based heat source in flow of Carreau fluid with cross-diffusion effects," Journal of NonEquilibrium Thermodynamics, vol. 44, no. 2, pp. 203-213, 2019.

[34] Z. Shafique, M. Mustafa, and A. Mushtaq, "Boundary layer flow of Maxwell fluid in rotating frame with binary chemical reaction and activation energy," Results in Physics, vol. 6, pp. 627-633, 2016. 